\title{
Current Trends in Management of Fetal Growth Restriction
}

\author{
Kanwal Gujral $\cdot$ Sakshi Nayar
}

Received: 5 January 2015/Revised: 3 February 2015/Accepted: 5 February 2015/Published online: 25 February 2015

(C) Society of Fetal Medicine 2015

\begin{abstract}
Fetal growth restriction (FGR) or intrauterine growth restriction (IUGR) are the terms used for a fetus which has not attained its full growth potential. FGR fetus is associated with high perinatal and neonatal morbidity and mortality, besides long term neurological handicap because of in utero hypoxia. Three decades of research has indicated that almost all treatment strategies are ineffective in treating FGR. Panorama of management includes identifying women at risk, correct dating, distinguishing between a constitutionally small and a true FGR, monitoring for fetal hypoxia, growth, and a timely intervention to avoid death or damage.
\end{abstract}

Keyword Fetal Growth Restriction - Intrauterine Fetal Growth Restriction · Intrauterine Growth Retardation

\section{Introduction}

Fetal growth restriction (FGR) or intrauterine growth restriction (IUGR) are the terms used for a fetus which has not attained its full growth potential, usually less than 10 centile at a frequency of $10 \%$ of all pregnancies [1]. FGR is associated with high perinatal and neonatal mortality and morbidity, besides long term neurological handicap. These babies also suffer from metabolic syndrome in adult life, a disease with its origin in utero.

K. Gujral ( $\square)$

Institute of Obstetrics \& Gynaecology, Sir Ganga Ram Hospital,

Rajinder Nagar, New Delhi 110060, India

e-mail: kgg_in@yahoo.com

S. Nayar

Department of Obstetrics \& Gynaecology, Lady Hardinge

Medical College \& Smt. S. K. Hospital, New Delhi, India
Disappointingly, treatment strategies are largely ineffective, therefore, the crux of the management is monitoring for fetal hypoxia and a timely intervention to deliver.

\section{Etiology}

Etiology may be due to maternal, fetal, or placental disorders [2]. These are set in Table 1.

\section{Nomenclature}

There is a change in thinking on the nomenclature of FGR. Symmetrical and asymmetrical terms are no longer used because majority of asymmetrical FGRs become symmetrical later and asymmetrical ones do not do better than symmetrical ones, unlike what was thought earlier. Currently, FGR is classified as early onset (onset $<34$ weeks gestation, usually starting in mid-trimester) and late onset (onset $\geq 34$ weeks gestation). Early onset FGR differs from late onset one in its response to hypoxia. Early onset FGR fetus responds to hypoxia/chronic deprivation in a hierarchical manner from compensatory to a decompensatory phase. These changes are depicted in Fig. 1 [3].

Usually, early onset FGR shows the above mentioned changes from arterial to venous circulation but at times, cardiovascular (Doppler) and behavioral response of fetus (BPP) can occur independent of each other resulting in discordant Doppler and BPP changes. Therefore, an integrated approach using multivessel Dopplers and BPP is the recommended management practice.

In late onset FGR, sequential changes from arterial to venous circulation are absent. Umbilical artery Doppler is usually normal in up to $20 \%$ of cases. Cerebral 
Table 1 Etiology of FGR

\begin{tabular}{ll}
\hline Conditions & Causes \\
\hline Maternal & Chronic hypertension, pregestational diabetes, cardiovascular disease, substance abuse, autoimmune conditions \\
Fetal & Infection, malformation, aneuploidy \\
Placental & Chorioangioma, infarction, circumvallate placenta, confined placental mosaicism, obliterative vasculopathy of placental bed
\end{tabular}

Decrease in umbilical venous volume \& liver size Abdominal circumference $(\mathrm{AC}) \mathrm{lag}-1^{\text {st }}$ biometric sign Increased umbilical artery doppler resistance Flow redistribution - Middle Cerebral Artery(MCA) compensatory vasodilatation

Decreased amniotic fluid

Absent end diastolic velocity (AEDV) in umbilical artery

Loss of fetal movement, variability

Reversed end diastolic flow in umbilical artery

Ductus venosus [a-wave absent or reversal]

Biophysical profile (BPP) or score (BPS) decline

non reactive NST, late decelerations

Fetal circulatory collapse \& death

Fig. 1 Sequence of changes in response to hypoxia/chronic deprivation of fetus

redistribution can occur with normal umbilical artery Doppler. Decreased cerebroplacental ratio (CPR) precedes middle cerebral artery (MCA) dilatation. Such a decrease should alert the obstetrician that brain sparing is imminent. Late onset FGR is a significant clinical problem that frequently goes undetected and can contribute to over $50 \%$ of unanticipated still births $[2,4]$.

\section{Management}

First step in the management is correct dating, for which known last menstrual period (LMP), regular cycles, clinical examination in early pregnancy, and most importantly, ultrasound dating in first or early second trimester, are the tools.

Second step is to establish the presence of FGR by using fetal weight or biometry less than 10th, 5th or 3rd centile preferably on a customized growth chart which takes into account fetal growth potential based upon mother's height, prepregnancy weight, parity and ethnicity. A 10th centile, cutoff is the usual practice in establishing FGR.

Third step is to differentiate between true growth restriction (FGR and abnormal Doppler flows) and constitutionally small fetus-small for gestation age (SGA) (FGR and normal Doppler flows). Fetuses at 3rd centile or less are usually true FGRs.
The Porto study [5] has indicated that between 3rd and 10th centile FGR incidence of adverse outcome is $2 \%$, at 3rd centile, it is $6.2 \%$ and if 3rd centile growth and Dopplers $>95$ th centile, absent or reverse flows in umbilical arteries, it is $16.7 \%$.

Fourth step FGR is established; viable gestation less than 34 weeks, careful search for fetal anomalies (ultrasonography and fetal echocardiography), and selective investigations for maternal and fetal causes should be done.

Ten per cent of FGRs have congenital malformations; $10-40 \%$ of structurally malformed fetuses will have chromosomal abnormalities as against only $2 \%$ of normally formed fetuses. Chromosomal studies in the fetus are recommended.

Work up for fetal infection should also be undertaken, if clinically suspected. Infections account for less than $5 \%$ of all FGRs.

Needless to say that underlying maternal disease such as hypertension disorders, autoimmune disease etc., if present, should be treated.

Fifth step is the most important step of management, that is, monitoring for fetal hypoxia. An integrated surveillance approach using multivessel Doppler-arterial and venous, BPS, and fetal growth assessment is the recommended practice. Figures 2, 3, 4 indicate a rough management protocol in different scenarios.

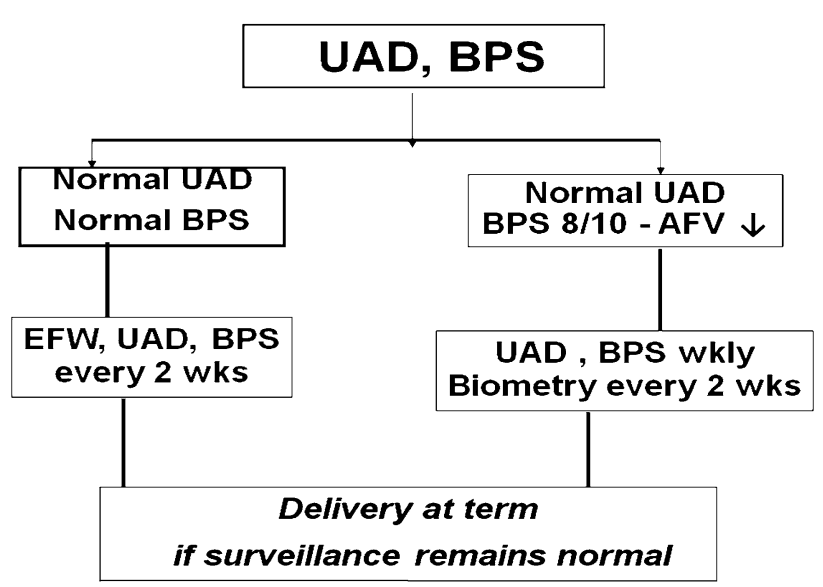

Fig. 2 Scenario 1. $A F V$ amniotic fluid volume, BPS biophysical score, $E F W$ expected fetal weight, $U A D$ umbilical artery Doppler 
Fig. 3 Scenario 2. $A F V$ amniotic fluid volume, $B P S$ biophysical score, $M C A$ middle cerebral artery, MCAPI middle cerebral artery pulsatility index, $U A D$ umbilical artery Doppler, UAPI umbilical artery pulsatility index

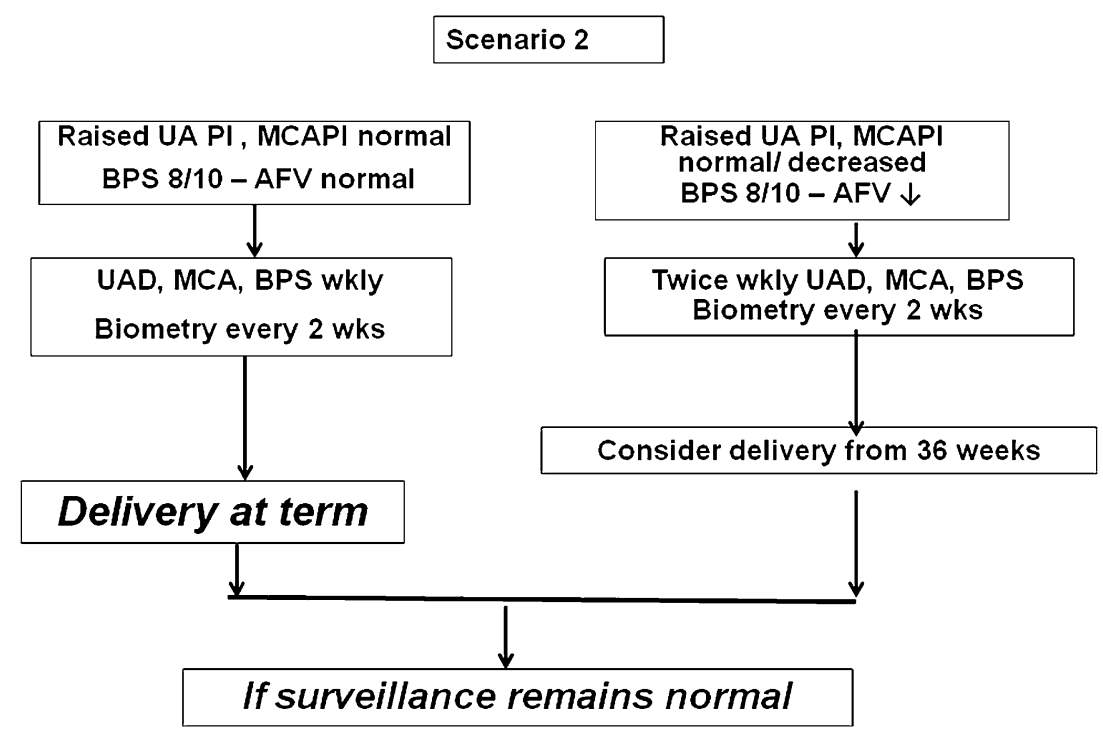

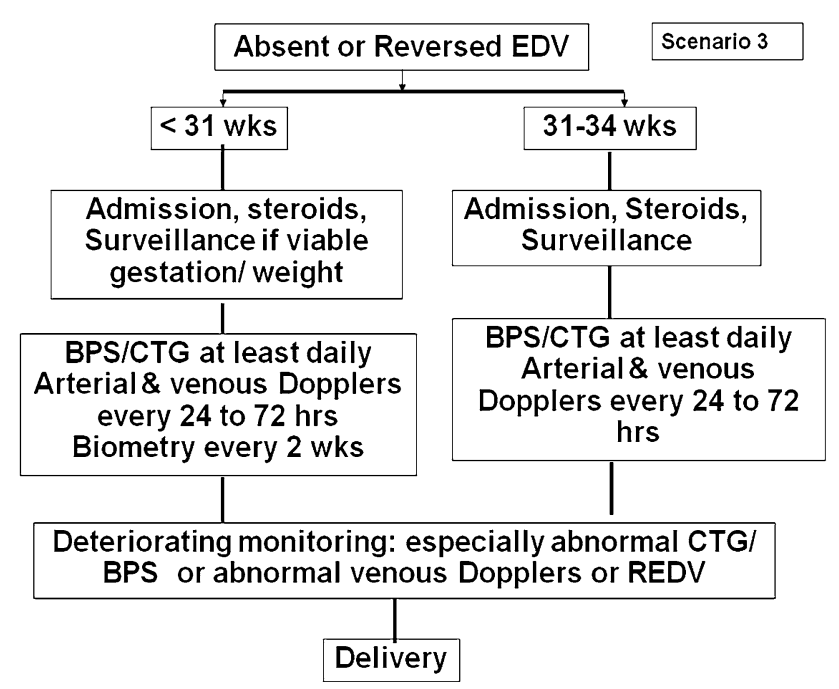

Fig. 4 Scenario 3. BPS biophysical score, CTG cardiotocography, REDV reverse end diastolic velocity

Scenario 1

\section{FGR Fetus with Normal Umbilical Artery Doppler} and BPS

This, most probably, is an SGA fetus that can be safely monitored every 2 weeks with umbilical artery Doppler, BPS and growth assessment. Pregnancy can be taken to term in the event of normal findings.

\section{FGR Fetus with Normal Umbilical Artery Doppler but Reduced Amniotic Fluid Volume}

This fetus needs weekly surveillance. Pregnancy can also be taken to term.

\section{Scenario 2}

FGR Fetus with Increased Umbilical Artery Resistance but not Absent Flow, Normal MCA Doppler and Normal Amniotic Fluid Volume

This case scenario depicts a fetus which is compromised but has not redistributed its cardiac output. Weekly monitoring is required with umbilical artery Doppler, MCA Doppler and BPS. Fetal growth check is recommended every 2 weeks. Pregnancy can be taken to term if surveillance remains normal.

FGR Fetus with Raised Umbilical Artery Doppler, Dilated Middle Cerebral Artery and Reduced Amniotic Fluid Volume

FGR fetus that has raised umbilical artery Doppler, dilated MCA and reduced amniotic fluid volume is a fetus which has redistributed its cardiac output, therefore has some degree of hypoxemia. Fetus must be monitored biweekly with a plan to deliver by 36 weeks.

Scenario 3

Fetus with Absent End Diastolic or Reverse Flow in Umbilical Arteries

When $70 \%$ of placental circulation is affected, absent or reverse flow in umbilical artery occurs. There is significant degree of hypoxemia. When venous circulation gets affected, acidemia is likely. Therefore, daily monitoring with arterial and venous Doppler is recommended, if the fetus is premature. Delivery is planned by $32-34$ weeks. 


\section{Timing of Delivery}

It is an important issue in the management of FGR. Delivery should be considered whenever risk of fetal death exceeds that of neonatal death. Gestational age and individual NICU facilities are important contributors to survival rates.

The growth restriction intervention trial (GRIT) $[6,7]$, while addressing the issue of immediate versus delayed delivery in severe FGRs, has indicated that early delivery group had fewer stillbirths but more neonatal deaths, whereas, delayed delivery resulted in some antepartum deaths. Long term outcomes at 13 years remained the same.

Generally, reverse end diastolic velocity (REDV) is delivered at 32 weeks, absent end diastolic velocity (AEDV) at 34 weeks and other FGRs with high umbilical artery Doppler indices but not absent and other tests remaining normal, are delivered at 37 weeks.

In general, whenever antepartum monitoring tests indicate imminent hypoxia, such as abnormal cardiotocography (CTG), BPS $<4$, severe oligoamnios, ductus venosus $\geq 95 \%$ and umbilical vein pulsations-fetus should be delivered. No interval growth in $2-3$ weeks, also, is an indication for delivery.

Regarding mode of delivery, cesarean section is the preferred mode because these fetuses are hypoxic, oligoamniotic and, therefore, withstand labor poorly.

Normal delivery can be done if cervix is ripe and facilities for continuous electronic fetal monitoring are available. It is advisable to send umbilical cord blood analysis to establish neonatal base line status.

For late onset FGR, monitoring twice or thrice a week with umbilical artery Doppler, MCA Doppler and CPR is recommended. Delivery should be considered at 37 weeks.

\section{Therapy for Treatment of FGR}

Many therapies have been tried. Bed rest, maternal nutrient supplements, calcium channel blockers, beta mimetics, maternal oxygenation, plasma volume expansion, and transcutaneous electrostimulation have not proven to have any benefit for treatment of FGR, by Cochrane systematic reviews [8-14].

Sildenafil citrate therapy for treatment of severe early onset FGR has been reported to have improved fetal growth in a recent study [15]. Strider trial, launched in 2014, will address the issue of sildenafil in improving outcomes, growth velocity, and reducing serious adverse outcomes in severely growth restricted fetuses [16].

Antenatal glucocorticoids confer similar benefits of reducing respiratory distress syndrome and its related morbidities, when given to preterm FGR fetuses, as they do in preterm adequate for gestational age fetuses. Three studies have shown that fetuses with AEDV often show a transient improvement in blood flow after glucocorticoid administration, and fetuses that did not show improvement appeared to have poorer outcomes [17-19].

Magnesium sulphate used prior to delivery in gestational ages $<34$ weeks reduces death, cerebral palsy, and gross motor dysfunctions in neonates [20]. The recommended dose is $4 \mathrm{~g}$ as a loading dose intravenously, followed by $1 \mathrm{~g} / \mathrm{h}$ for a maximum of $24 \mathrm{~h}$.

\section{Antithrombotic Therapy and FGR}

Asprin

When started at an early gestational age ( $<16$ weeks), asprin has shown to reduce perinatal death, pre-eclampsia and adverse outcome in women who are at risk of developing FGR [21-23]. In fact, Royal College of Obstetricians and Gynecologists (RCOG) suggests screening for FGR in early pregnancy by risk factors, and, in the presence of three minor or one major, asprin prophylaxis is recommended [24].

\section{Heparin}

A recent Cochrane review has suggested that use of heparin also appears to be associated with a statistically significant reduction in risk of perinatal mortality, preterm birth before 34 weeks and infant birth weight below 10th centile for gestational age in women considered at risk of placental dysfunction [25].

\section{Conclusion}

Optimal management of suspected FGR involves identifying women at risk, correct dating, distinguishing between constitutionally small and true FGRs, monitoring for fetal well being, growth, and a timely intervention.

Conflict of interest None.

\section{References}

1. Vandenbosche RC, Kirchner JT. Intrauterine growth retardation. Am Fam Physician. 1998;58(6):1384-90.

2. Vladareanu R, Burnei A, Constantinescu S. Timing and mode of delivery of the infant with IUGR. Curr Prog Obstet Gynecol. $2014 ; 2: 135-50$. 
3. Baschat AA. Fetal growth restriction: from observation to intervention. J Perinat Med. 2010;38(3):239-46.

4. Oros D, Figueras F, Cruz-Martinez R, Meler E, Munmany M, Gratacos E. Longitudinal changes in uterine, umbilical and fetal cerebral Doppler indices in late-onset small-for-gestational age fetuses. Ultrasound Obstet Gynecol. 2011;37:191-5.

5. Unterscheider J, Daly S, Geary MP, Mairead MK, McAuliffe FM, O'Donoghue $\mathrm{K}$, et al. Optimizing the definition of intrauterine growth restriction: the multicenter prospective PORTO Study. Am J Obstet Gynecol. 2013;208(290):e1-6.

6. The GRIT Study Group. When do obstetricians recommend delivery for a high-risk preterm growth-retarded fetus? Growth restriction intervention trial. Eur J Obstet Gynecol Reprod Biol. 1996;67:121-6.

7. Thornton JG, Hornbuckle J, Vail A, Spiegelhalter DJ, Levene M. Infant wellbeing at 2 years of age in the Growth Restriction Intervention Trial (GRIT): multicentred randomised controlled trial. Lancet. 2004;364:513-20.

8. Say L, Gülmezoglu AM, Hofmeyr GJ. Bed rest in hospital for suspected impaired fetal growth. Cochrane Database of Systematic Reviews 1996, Issue 1. Art. No.: CD000034.The Cochrane Library 2010, Issue 7. Updated 2010, Issue 7.

9. Say L, Gülmezoglu AM, Hofmeyr GJ. Maternal nutrient supplementation for suspected impaired fetal growth (Review). Cochrane Database Syst Rev 2003, Issue 1. Art. No.: CD000148. doi: 10.1002/14651858.CD000148. Updated 2010, Issue 7.

10. Say L, Gülmezoglu AM, Hofmeyr GJ. Calcium channel blockers for potential impaired fetal growth. Cochrane Database Syst Rev 1996, Issue 1. Art. No.: CD000049. doi: 10.1002/14651858. CD000049. Updated 2009, Issue 4.

11. Say L, Gülmezoglu AM, Hofmeyr GJ. Betamimetics for suspected impaired fetal growth. Cochrane Database Syst Rev 2001, Issue 4. Art. No.: CD000036. doi: 10.1002/14651858.CD000036. Updated 2009, Issue 4.

12. Say L, Gülmezoglu AM, Hofmeyr GJ. Maternal oxygen administration for suspected impaired fetal growth. Cochrane Database Syst Rev 2003, Issue 1. Art. No.: CD000137. doi: 10.1002/ 14651858.CD000137. Updated 2009, Issue 4.

13. Say L, Gülmezoglu AM, Hofmeyr GJ. Plasma volume expansion for suspected impaired fetal growth.. Cochrane Database Syst Rev 1996, Issue 4. Art. No.: CD000167. doi: 10.1002/14651858. CD000167. Updated 2010, Issue 7.

14. Say L, Gülmezoglu AM, Hofmeyr GJ. Transcutaneous electrostimulation for suspected placental insufficiency (diagnosed by Doppler studies). Cochrane Database Syst Rev 1996, Issue 1. Art. No.: CD000079. doi: 10.1002/14651858.CD000079. Updated 2009, Issue 4.
15. Von Dadelszen P, Dwinnell S, Magee LA, Carleton BC, Gruslin A, Lee B, et al. Research into Advanced Fetal Diagnosis and Therapy (RAFT) Group. Sildenafil citrate therapy for severe early-onset intrauterine growth restriction. BJOG. 2011;118(5):624-8.

16. Ganzevoort W, Alfirevic Z, von Dadelszen P, Kenny L, Papageorghiou A, van Wassenaer-Leemhuis A, et al. STRIDER: sildenafil therapy in dismal prognosis early-onset intrauterine growth restriction-a protocol for a systematic review with individual participant data and aggregate data meta-analysis and trial sequential analysis. Syst Rev. 2014;11:3-23.

17. Robertson MC, Murila F, Tong S, Baker LS, Yu VY, Wallace EM. Predicting perinatal outcome through changes in umbilical artery Doppler studies after antenatal corticosteroids in the growth-restricted fetus. Obstet Gynecol. 2009;113:636-40.

18. Simchen MJ, Alkazaleh F, Adamson SL, Windrim R, Telford J, Beyene $\mathrm{J}$, et al. The fetal cardiovascular response to antenatal steroids in severe early-onset intrauterine growth restriction. Am J Obstet Gynecol. 2004;190:296-304.

19. Nozaki AM, Francisco RP, Fonseca ES, Miyadahira S, Zugaib M. Fetal hemodynamic changes following maternal betamethasone administration in pregnancies with fetal growth restriction and absent end-diastolic flow in the umbilical artery. Acta Obstet Gynecol Scand. 2009;88:350.

20. Doyle LW, Crowther CA, Middleton R. Magnessium sulphate for women at risk of preterm births for neuroprotection of the fetus. Cochrane Database Syst Rev 2009;CD004661.

21. Uzan S, Beaufils M, Breart G, Bazin B, Capitant C, Paris J. Prevention of fetal growth retardation with low-dose aspirin: findings of the EPREDA trial. Lancet. 1991;337:1427-31.

22. Leitich H, Egarter C, Husslein P, Kaider A, Schemper M. A meta-analysis of low dose aspirin for the prevention of intrauterine growth retardation. $\mathrm{Br} \mathrm{J}$ Obstet Gynaecol. 1997;104(4):450-9.

23. Roberge S, Nicolaides KH, Demers S, Vlla P, Bujold E. Prevention of perinatal death and adverse perinatal outcome using low-dose aspirin: a meta-analysis. Ultrasound Obstet Gynecol. 2013;41(5):491-9.

24. The investigation and management of the small for gestational age fetus. RCOG, Green top Guideline No.31. 2nd Ed. February 2013, Minor revision January 2014.

25. Dodd JM, McLeod A, Windrim RC, Kingdom J. Antithrombotic therapy for improving maternal or infant health outcomes in women considered at risk of placental dysfunction. Cochrane Database Syst Rev. 2013, Issue 7. 